\title{
Bandwidth-length trade-off figures of merit for electro-optic traveling wave modulators
}

\author{
Jose A. Ibarra Fuste* and Maria C. Santos Blanco \\ Signal Theory and Communications Department, Universitat Politecnica de Catalunya, Campus Nord UPC, \\ Building D3-114, Jordi Girona St., 1-3, Barcelona 08034, Spain \\ ${ }^{*}$ Corresponding author: ibarra@tsc.upc.edu
}

Received February 25, 2013; accepted March 25, 2013;

posted April 3, 2013 (Doc. ID 185869); published April 30, 2013

\begin{abstract}
Closed-form expressions explicitly relating modulation bandwidth and active length in electro-optic traveling wave modulators are presented which fully account for skin-effect electrode loss and optical-electrical wave velocities mismatch. Four operative margins have been identified where the bandwidth-length trade-off figure of merit takes simple forms. (C) 2013 Optical Society of America
\end{abstract}

OCIS codes: (230.2090) Electro-optical devices; (250.4110) Modulators.

http://dx.doi.org/10.1364/OL.38.001548

Optical modulators based on the electro-optic (EO) Pockels effect are basic building blocks of optical systems, with applications continuously growing up. As compared to lumped electrode configurations, bounded by a fundamental sensitivity-bandwidth limit, traveling wave (TW) configurations allow pushing the limit by optimization of the TW structure and provide extended modulation bandwidth with reduced drive power $[1,2]$.

In EO-TW modulators (EO-TWM) the Pockels electrically induced optical phase shift accumulates with the copropagated distance (L) and therefore, the modulation drive voltage, usually quantified as the voltage required for a $\pi$ phase shift, $V_{\pi}$, is reduced proportionally to increases in L [3]. However, as it is well known, this comes at the expense of a corresponding reduction in operative bandwidth (B), which in typical EO-TWM based on coplanar waveguides (CPWs) over $\mathrm{LiNbO}_{3}$ substrates stems mainly from the combined action of two basic mechanisms, namely the skin-effect electrode loss and the optical-electrical wave velocity mismatch. When trying to elucidate the expected $B$ reduction that a specific $L$ increase could have, a constant $B L$ product rule proportional to the inverse of the velocity matching (VM) constant $(\nu)$ has been shown to govern the low-loss (LL) limit [4], while in the VM limit, a constant $B L^{2}$ rule proportional to the inverse of the square of the loss constant $(\alpha)$ has been found more appropriate [5]. To the best of our knowledge, no simple B-L rules have been derived for the intermediate ranges in which both $\alpha$ and $\nu$ are relevant, nor have the LL and VM limits been quantified in a general way.

In this Letter we present closed-form expressions that fully account for the effects of skin-effect electrode loss and optical-electrical wave velocities mismatch and that explicitly and in a biunivocal way relate the operative bandwidth and the electrode length in EO-TWM. From these, four B-L trade-off figures of merit are identified with different validity ranges which are seen to depend both on the TW cross-sectional parameters (i.e., skineffect constant and optical-electrical velocity mismatch) and on the target $\mathrm{B}$ and $\mathrm{L}$ values.

In order to analyze the $\mathrm{B}-\mathrm{L}$ relationship, we begin by considering the electrical modulation frequency response of an impedance matched EO-TWM in the presence of skin-effect electrical loss in the conducting electrodes of length $L$ and optical-electrical velocity mismatch [ $[6]$

$$
M(f)=e^{-\frac{\alpha_{0} \sqrt{f L}}{2}}\left[\frac{\sinh ^{2}\left(\frac{\alpha_{0} \sqrt{f} L}{2}\right)+\sin ^{2}(\pi f L L)}{\left(\frac{\alpha_{0} \sqrt{f} L}{2}\right)^{2}+(\pi f \nu L)^{2}}\right]^{1 / 2},
$$

where $\alpha_{0}$ is the field attenuation coefficient in Nepers per unit length at $1 \mathrm{GHz}, \nu=\left(n_{m}-n_{\text {opt }}\right) / c$ is the velocity mismatch parameter, with $c$ the speed of light in vacuum, and $n_{m}$ and $n_{\text {opt }}$ the group effective refractive indices of the microwave and optical waves, respectively.

In order to maintain broadband operation of the modulator, the electrode design usually puts an emphasis in achieving impedance matching to driving sources. Residual impedance mismatches may simply add a loss factor in Eq. (1) with a small frequency dependence in the majority of cases. An analysis of the influence of impedance mismatch can be found in [7].

For the sake of completeness, and also to be able to compare with reported experiments, we will be giving general expressions for both the electrical and optical bandwidths verifying, respectively, []

$$
M\left(B^{e}\right)=1 / \sqrt{2}, \quad M\left(B^{o}\right)=1 / 2 .
$$

The LL and VM cases are simply obtained by taking limits in (1), as by $[\underline{4}, \underline{5}, \underline{9}]$

$$
\begin{array}{ll}
B^{(e, o)}=\frac{k_{\nu}^{(e, o)}}{\nu L} ; & \alpha_{0} \rightarrow 0, \\
B^{(e, o)}=\frac{k_{\alpha}^{(e, o)}}{\alpha_{0}^{2} L^{2}} ; & \nu \rightarrow 0,
\end{array}
$$

where the constants $k_{\nu}^{(e)}=0.4429, \quad k_{\nu}^{(o)}=0.6033$, $k_{\alpha}^{(e)}=0.5452, k_{\alpha}^{(o)}=2.5396$, when $B$ is given in GHz, $L$ in $\mathrm{cm}, \nu$ in $\mathrm{ns} / \mathrm{cm}$ and $\alpha_{0}$ in $N p /(\mathrm{cm} \sqrt{\mathrm{GHz}})$.

From now on, we drop the super indexes indicating electrical or optical bandwidth, as the results are interchangeable by only using in the expressions the corresponding values of $k_{\nu}$ and $k_{\alpha}$. 
In a $\alpha_{0}-\nu$ plane connecting together the points yielding the same $B$ and $L$ values, curves with parabolic-like patterns are formed. As it follows from Eq. (3), these curves intersect the $\nu$ axis in the $k_{\nu} / B L$ point, and the $\alpha_{0}$ axis in the $\sqrt{k_{\alpha}} / \sqrt{B} L$ point; by numerical curve fitting we have found that they are well described by

$$
B^{2} L^{2}+\frac{k_{\nu}^{2}}{\sqrt{k_{\alpha}}} \frac{\alpha_{0}}{\nu^{2}} \sqrt{B} L=\frac{k_{\nu}^{2}}{\nu^{2}}
$$

Numerical solutions to Eq. (2) are seen to fulfill the above expression with accuracy in the range of the numerical error margin.

Definition of reference bandwidth and length values for the modulator as follows:

$$
\begin{gathered}
B_{M}=\frac{k_{\nu}^{2}}{k_{\alpha}} \frac{\alpha_{0}^{2}}{\nu^{2}}, \\
L_{M}=\frac{1}{2} \frac{k_{\alpha}}{k_{\nu}} \frac{\nu}{\alpha_{0}^{2}},
\end{gathered}
$$

allows to write the analytic solutions for $B$ and $L$ in Eq. (4) in a convenient normalized form

$$
\begin{gathered}
B_{N}=\frac{B}{B_{M}}=\left(2 L_{N}\right)^{-2 / 3} T\left[1-\sqrt{T^{-3 / 2}-1}\right]^{2}, \\
L_{N}=\frac{L}{L_{M}}=B_{N}^{-3 / 2}\left[\sqrt{4 B_{N}+1}-1\right],
\end{gathered}
$$

where

$$
\begin{gathered}
T=\frac{1}{2} W^{-1 / 3}\left(S^{1 / 3}-S^{-1 / 3}\right), \\
S=W+\sqrt{1+W^{2}}, \\
W=\frac{3^{3 / 2}}{2^{5}} L_{N} .
\end{gathered}
$$

Equations (7) and (8) provide a useful analytical tool to calculate $\mathrm{B}$ and $\mathrm{L}$ for every specific TW configuration defined by its cross-sectional parameters $\alpha_{0}$ and $\nu$. Additionally, they allow to build a normalized $B_{N}-L_{N}$ chart such as that in Fig. 1, where the LL and VM operative ranges, characterized by the $B L$ and $B L^{2}$ trade-off rules, respectively, are clearly distinguished. As shown, the reference $B_{M}$ and $L_{M}$ values can be advantageously used to express the trade-off figures of merit in a compact and easy to remember way as $B L=2 B_{M} L_{M}$ and $B L^{2}=4 B_{M} L_{M}^{2}$, respectively.

Two additional trade-off ranges, quasi-LL (QLL) and quasi-VM (QVM), have been identified in which the applicable trade-off figures of merit can be given as $B L^{7 / 6}=$ $5 / 4 B_{M} L_{M}^{7 / 6}$, and $B L^{5 / 3}=7 / 4 B_{M} L_{M}^{5 / 3}$, respectively.

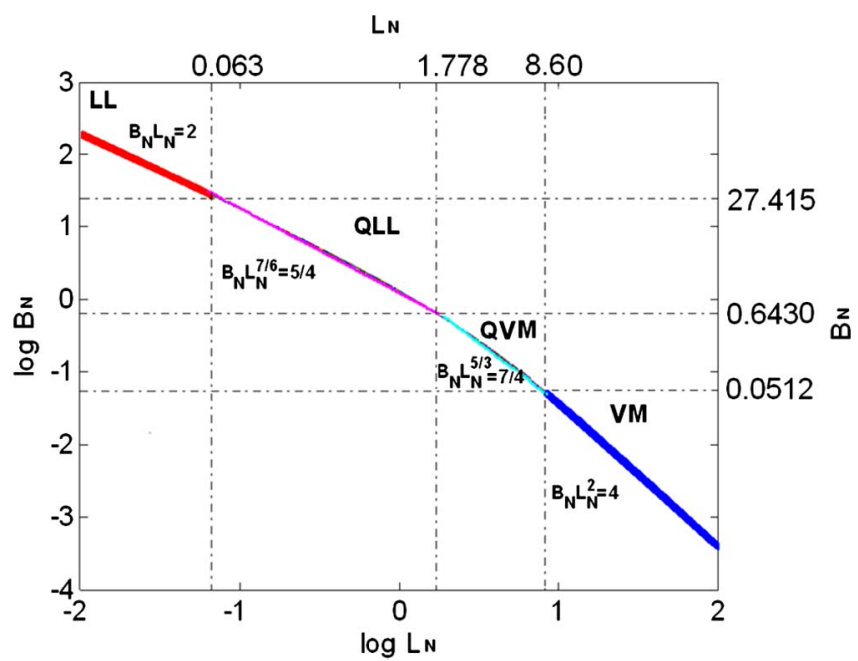

Fig. 1. Normalized $B_{N}-L_{N}$ chart.

The limiting $B_{N}$ and $L_{N}$ values for a calculated $10 \%$ maximum departure of the trade-off rule against numerical solutions to Eq. (2) are also given in the chart for reference.

It is worth noting that operation into a specific tradeoff margin (LL, QLL, QVM, VM) is dependent not only on the values of $\alpha_{0}$ and $\nu$, but also on the target $B-L$ values of every specific application. Thus, one can always work into the VM margin for example, regardless of the crosssectional configuration with only choosing to work with a long enough $L$ /narrow enough $B\left(L>8.60 L_{M}\right.$, $B<0.0512 B_{M}$ ), provided of course that the values obtained have a physical relevance and guarantee the applicability of the approximations of the model.

This finding is in accordance with the work of Chowdhury and McCaughan [5] who concluded that the VM figure of merit, for a given $\alpha_{0}=6.9 \mathrm{~Np} / \mathrm{m}-\sqrt{\mathrm{GHz}}$ and $\left|N_{m}-N_{o}\right| \leq 0.03$, was valid for a $B<30 \mathrm{GHz}$ and $L>4.7 \mathrm{~cm}$. By applying the general expression in our model to those specific values of $\alpha_{0}$ and $\nu$, we obtain $B<0.0512 B_{M}=35 \mathrm{GHz}$ and $L>8.60 L_{M}=3.8 \mathrm{~cm}$.

Next, we applied the trade-off rules to $4 \mathrm{LiNbO}_{3} \mathrm{EO}-$ TWM with CPW electrodes extracted from the literature. Table 1 shows the results obtained. From the $\alpha_{0}-\nu$ parameters and the crystal cut length of each design, we have determined the relevant trade-off margin using the limits in the $B_{N}-L_{N}$ chart, and we have listed also the bandwidth as measured, as given by the complete closed form Eq. (7), and as approximated using the corresponding trade-off rule. Depending on the specific definition of bandwidth used in each reference, optical $(o)$ or electrical $(e)$, the right $k_{\nu}$ and $k_{\alpha}$ constants had

Table 1. Parameters of the Reference Structures

\begin{tabular}{lcccc}
\hline Ref. & $\underline{\underline{10}}]$ & {$[\underline{11}]$} & {$[\underline{\underline{12}}]$} & $\underline{[13}]$ \\
\hline$\alpha_{0}(N p / \mathrm{cm} \sqrt{\mathrm{GHz}})$ & 0.0288 & 0.0864 & 0.1046 & 0.0461 \\
$\nu(\mathrm{ns} / \mathrm{cm})$ & 0.0050 & 0.0017 & 0.0043 & 0.0033 \\
$L(\mathrm{~cm})$ & 3.5 & 2.7 & 2.5 & 4 \\
trade-off & $\mathrm{QLL}$ & $\mathrm{QLL}$ & $\mathrm{QVM}$ & $\mathrm{QLL}$ \\
$B(\mathrm{GHz})$ (meas.) & $14(e)$ & $40(o)$ & $23(o)$ & $28(o)$ \\
$B(\mathrm{GHz})$ (Eq. $)$ & $16.8(e)$ & $39(o)$ & $24.4(o)$ & $28.3(o)$ \\
$B(\mathrm{GHz})$ (rule) & $16(e)$ & $36.5(o)$ & $23.5(o)$ & $27.6(o)$ \\
\hline
\end{tabular}


to be used in the reference length and bandwidth definitions in Eqs. (5) and (ㅁ).

As seen, agreements better than $20 \%$ confirm that the analytical model here described is well suited to assessment of the bandwidth-length trade-off in EO-TWM.

In summary, explicit closed form expressions, relating bandwidth and length in traveling-wave EO modulators limited by the skin-effect electrode losses and opticalelectrical wave velocity mismatch, have been presented. The expressions are given in a normalized way providing a unified framework for the analysis of any cross-sectional TW structure. A normalized B to L chart derived from the expressions offers an insightful and complete picture of the B to L trade-off and clearly shows four operative margins where the $\mathrm{B}$ to $\mathrm{L}$ figure of merit takes simple forms.

Definition of reference $B$ and $L$ values as a function of the TW cross-sectional parameters allows for compact expressions and for assessment of the potential of each TW cross-section in terms of achievable B and L values. The model here presented thus shows promise to constitute a powerful tool in advancing the performance of E0-TWM, which is the key to the progress of optical systems industry.

This work was supported in part by the Spanish CICYT projects TEC2011-25215 and TEC2012-3479914.

\section{References}

1. L. Wooten, K. Kissa, A. Yi-Yam, E. Murphy, D. Lafaw, P. Hallemeir, D. Maack, D. Attanasio, D. Fritz, G. McBrien, and D. Bossi, IEEE J. Sel. Top. Quantum Electron 6, 69 (2000).

2. R. C. Alferness, IEEE Trans. Microwave Theory Tech. 30, 1121 (1982).

3. B. E. A. Saleh and M. C. Teich, Fundamental of Photonics (Wiley, 1991).

4. R. Alferness, S. Korotky, and E. Marcatili, IEEE J. Quantum Electron. 6, 69 (1984).

5. A. Chowdhury and L. McCaughan, Opt. Lett. 26, 1317 (2001).

6. D. Janner, D. Tulli, M. Belmonte, and V. Pruneri, J. Opt. A 10, 1 (2008).

7. S. Haxha, M. A. Rahman, and K. T. V. Grattan, Appl. Opt. 42, 2674 (2003).

8. O. Mitomi, K. Noguchi, and H. Miyazaka, IEEE Proc. Optoelectron. 145, 360 (1998).

9. K. Noguchi, O. Mitomi, and H. Miyazawa, J. Lightwave Technol. 16, 615 (1998).

10. K. Aoki, J. Kondo, Y. Iwata, A. Hamajima, T. Ejiri, O. Mitomi, and M. Minakata, in 2006 European Conference on Optical Communications (ECOC, 2006), p. 1.

11. K. Noguchi, O. Mitomi, K. Kawano, and M. Yanagibashi, IEEE Photon. Technol. Lett. 5, 52 (1993).

12. M. Rangaraj, T. Hosoi, and M. Kondo, IEEE Photon. Technol. Lett. 4, 1020 (1992).

13. M. Minakata, Proc. SPIE 4532, 16 (2001). 